\title{
Rational Solutions to the ABS List: Transformation Approach
}

Danda ZHANG and Da-Jun ZHANG

Department of Mathematics, Shanghai University, Shanghai 200444, P.R. China

E-mail: zhangdd@shu.edu.cn,djzhang@staff.shu.edu.cn

Received March 21, 2017, in final form September 26, 2017; Published online October 02, 2017

https://doi.org/10.3842/SIGMA.2017.078

\begin{abstract}
In the paper we derive rational solutions for the lattice potential modified Korteweg-de Vries equation, and Q2, Q1 $(\delta), \mathrm{H} 3(\delta), \mathrm{H} 2$ and H1 in the Adler-Bobenko-Suris list. Bäcklund transformations between these lattice equations are used. All these rational solutions are related to a unified $\tau$ function in Casoratian form which obeys a bilinear superposition formula.
\end{abstract}

Key words: rational solutions; Bäcklund transformation; Casoratian; ABS list

2010 Mathematics Subject Classification: 35Q51; 35Q55

\section{Introduction}

In recent decades the research of discrete integrable systems has undergone rapid progress (see [13] and the references therein). As a new concept, multidimensional consistency, allowing suitable lattice equations to be embedded into a higher-dimensional space in a consistent way, has played an important role in the research of quadrilateral equations $[2,3,8,12,18,20]$. Quadrilateral equations that are consistent around the cube (CAC) with additional restriction ( $D_{4}$ symmetry and tetrahedron property) were searched and classified by Adler, Bobenko and Suris (ABS) [2] and in their list only 9 equations are included: Q4, Q3 $(\delta)$, Q2, Q1 $(\delta)$, A2, $\mathrm{A} 1(\delta), \mathrm{H} 3(\delta), \mathrm{H} 2$ and $\mathrm{H} 1$. All these equations have been solved from different approaches $[5,6,7,14,19,21,22]$.

As for rational solutions, which are solutions expressed by fractions of polynomials, in general, such type of solutions can be derived from soliton solutions through a special limit procedure (or a Taylor expansion), which corresponds to a way to generate multiple zero eigenvalues for certain spectral problems (see $[1,16]$ as examples). For the $\delta$-dependent equations in the ABS list, for example, $\mathrm{H} 3(\delta)$ and $\mathrm{Q} 1(\delta)$, the existence of $\delta$ (i.e., $\delta \neq 0$ ) plays a crucial role [21] in the procedure of obtaining rational solutions from their soliton solutions. For H1 which is independent of $\delta$, its rational solutions were obtained recently by making use of the HirotaMiwa equation and a continuous auxiliary variable [9]. Besides, as a generic $(2+1)-\mathrm{D}$ bilinear model, polynomial solutions of the Hirota-Miwa equation have been derived from several ways and presented via different forms $[11,17]$.

In this paper we systematically construct rational solutions for the ABS list by means of Bäcklund transformations (BTs). A fundamental role playing in the paper is the lattice potential modified Korteweg-de Vries (lpmKdV) equation. There is a non-auto BT which connects the lpmKdV equation and Q1(0) (also known as the lattice Schwarzian Korteweg-de Vries equation and cross-ratio equation). The two equations and their BT constitute a consistent triplet, say, viewing the BT as a two-component system, then the compatibility of each component yields

This paper is a contribution to the Special Issue on Symmetries and Integrability of Difference Equations. The full collection is available at http://www.emis.de/journals/SIGMA/SIDE12.html 
a lattice equation of another component which is in the triplet. This means any pair of solutions of the BT provide solutions to the two equations that the BT connects. Details will be shown in Sections 3.1 and 3.2 on how such a consistent triplet works in generating rational solutions. We also make use of non-auto BTs between equations in the ABS list [4]. Starting from the lpmKdV equation and Q1(0), rational solutions of Q2, Q1 $(\delta), \mathrm{A} 1(\delta), \mathrm{H} 3(\delta), \mathrm{H} 2$ and $\mathrm{H} 1$ in the ABS list can be derived through the map:

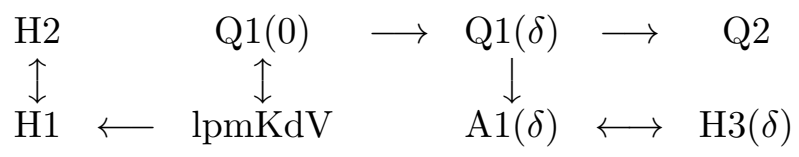

Figure 1. A map for generating rational solutions.

In the map the double-head arrow means the two equations it connects and their BT form a consistent triplet.

Moreover, we find all the obtained rational solutions are related to a unified $\tau$ function in Casoratian form which obeys a bilinear superposition formula (see (5.22)). Compared with those rational solutions of $\mathrm{H} 3(\delta)$ and $\mathrm{Q} 1(\delta)$ derived in [21], here we obtain new solutions. In fact, we will see that rational solutions of Q1 $(\delta)$ can explicitly be expressed through the rational solutions of Q1(0). Similar results hold for H3( $\delta)$ as well.

The paper is organized as follows. In Section 2 as preliminary we list quadrilateral equations that we consider in the paper and some notations. Then in Sections 3 and 4 we derive some rational solutions for the equations listed in Section 2. In Section 5 rational solutions in Casoratian form are proved. Finally in Section 6 we give conclusions.

\section{Preliminary}

We list quadrilateral equations that we consider in the paper:

$$
\begin{array}{ll}
\text { H1: } & (\widetilde{u}-\widehat{u})(\widehat{\widetilde{u}}-u)=q-p, \\
\mathrm{H} 2: & (\widetilde{v}-\widehat{v})(v-\widehat{\widetilde{v}})+(q-p)(v+\widetilde{v}+\widehat{v}+\widehat{\widetilde{v}})+q^{2}-p^{2}=0, \\
\text { lpmKdV: } & a(V \widetilde{V}-\widehat{\widetilde{V}})-b(V \widehat{V}-\widetilde{V} \widehat{\widetilde{V}})=0, \\
\mathrm{H} 3: & a(Z \widetilde{Z}+\widehat{Z} \widehat{\widetilde{Z}})-b(Z \widehat{Z}+\widetilde{Z} \widetilde{\widetilde{Z}})+2 \delta\left(a^{2}-b^{2}\right)=0, \\
\mathrm{Q} 1(0): & p(v-\widehat{v})(\widetilde{v}-\widehat{\widetilde{v}})-q(v-\widetilde{v})(\widehat{v}-\widehat{\widetilde{v}})=0, \\
\text { Q1 }(\delta): & p(u-\widehat{u})(\widetilde{u}-\widehat{\widetilde{u}})-q(u-\widetilde{u})(\widehat{u}-\widehat{\widetilde{u}})+\delta^{2} p q(p-q)=0, \\
\mathrm{~A} 1(\delta): & p(z+\widehat{z})(\widetilde{z}+\widehat{\widetilde{z}})-q(z+\widetilde{z})(\widehat{z}+\widehat{\widetilde{z}})-\delta^{2} p q(p-q)=0, \\
\mathrm{Q} 2: & p(w-\widehat{w})(\widetilde{w}-\widehat{\widetilde{w}})-q(w-\widetilde{w})(\widehat{w}-\widehat{\widetilde{w}})+p q(p-q)(w+\widetilde{w}+\widehat{w}+\widehat{\widetilde{w}}) \\
& \quad-p q(p-q)\left(p^{2}-p q+q^{2}\right)=0 .
\end{array}
$$

Here we use conventional notations $\widetilde{u} \doteq u_{n+1, m}, \widehat{u} \doteq u_{n, m+1}$. In the above equations, $p$ and $a$ are spacing parameters of $n$-direction and $q$ and $b$ are of $m$-direction; $\delta$ is an arbitrary constant.

Casoratian is a discrete version of Wronskian. Suppose that a basic column vector is

$$
\psi(n, m, l)=\left(\psi_{1}(n, m, l), \psi_{2}(n, m, l), \ldots, \psi_{N}(n, m, l)\right)^{\mathrm{T}} .
$$

Introduce a shift operator $E_{\nu}$ to denote

$$
E_{n}^{s} \psi \equiv \psi(n+s, m, l), \quad E_{m}^{s} \psi \equiv \psi(n, m+s, l), \quad E_{l}^{s} \psi \equiv \psi(n, m, l+s) .
$$


Then a $N$ th-order Casoratian w.r.t. $E_{l}$-shift is defined by

$$
\left|\psi, E_{l} \psi, E_{l}^{2} \psi, E_{l}^{3} \psi, \ldots, E_{l}^{N-1} \psi\right|
$$

and usually is compactly written as (cf. [10])

$$
|\widehat{N-1}|=|0,1,2, \ldots, N-1| \text {. }
$$

Another notation which is often used is $|\widehat{N-2}, N|=|0,1, \ldots, N-2, N|$.

Besides, in Wronskian/Casoratian verification of solutions to a bilinear equation, the equation is usually reduced to a Laplace expansion of a zero-valued $2 N \times 2 N$ determinant. The expansion is described as

Lemma 2.1 ([10]). Suppose that $\mathbf{B}$ is an $N \times(N-2)$ matrix and $\mathbf{a}, \mathbf{b}, \mathbf{c}, \mathbf{d}$ are $N$ th-order column vectors, then

$$
|\mathbf{B}, \mathbf{a}, \mathbf{b}||\mathbf{B}, \mathbf{c}, \mathbf{d}|-|\mathbf{B}, \mathbf{a}, \mathbf{c}||\mathbf{B}, \mathbf{b}, \mathbf{d}|+|\mathbf{B}, \mathbf{a}, \mathbf{d}||\mathbf{B}, \mathbf{b}, \mathbf{c}|=0 .
$$

\section{Rational solutions to lpmKdV, Q1, H3 and Q2}

In this section we first investigate relation between the lpmKdV equation and Q1(0). Such a relation will be used to construct rational solutions to not only the two equations themselves but also to $\mathrm{Q} 1(\delta), \mathrm{H} 3(\delta)$ and $\mathrm{Q} 2$.

\subsection{Solution sequence of $\mathrm{Q1}(0)$ and $\mathrm{lpmKdV}$}

Q1(0) is the equation (2.6) with $\delta=0$. Between (2.5) and the lpmKdV equation (2.3) there is a non-auto Bäcklund transformation [19]

$$
\widetilde{v}-v=a V \widetilde{V}, \quad \widehat{v}-v=b V \widehat{V},
$$

where

$$
p=a^{2}, \quad q=b^{2} .
$$

Equations (2.5), (2.3) and (3.1) constitute a consistent triplet in the following sense: as an equation set, the compatibility of $\widetilde{\widehat{v}}=\widehat{\widetilde{v}}$ and $\widetilde{\widehat{V}}=\widehat{\widetilde{V}}$ respectively yield (2.3) and (2.5).

Such an consistency can be used to construct solutions for equation (2.5) and (2.3):

Lemma 3.1. With the consistent triplet composed of (2.5), (2.3) and (3.1), we have the following:

(1) starting from any solution $v$ of (2.5), by integration through (3.1), the resulted $V$ solves equation (2.3), and vice versa;

(2) any solution pair $(v, V)$ of (3.1) gives a solution $v$ to (2.5) and $V$ to (2.3).

Further than that, we have

Lemma 3.2. For an arbitrary solution pair $(v, V)$ of (3.1) where $V \neq 0$, function $V_{1}=v / V$ solves the lpmKdV equation (2.3).

Proof. Substituting $V_{1}=v / V$ into (2.3) and making use of relation (3.1), it is easy to check $V_{1}$ satisfies (2.3). 
This lemma provides an approach to generate a sequence of solution pairs of the BT (3.1).

Theorem 3.3. For any solution pair $\left(v_{N}, V_{N}\right)$ of the BT (3.1), define

$$
V_{N+1}=\frac{v_{N}}{V_{N}}
$$

and it solves the lpmKdV equation (2.3). Next, the BT system

$$
\widetilde{v}_{N+1}-v_{N+1}=a V_{N+1} \widetilde{V}_{N+1}, \quad \widehat{v}_{N+1}-v_{N+1}=b V_{N+1} \widehat{V}_{N+1},
$$

(i.e., (3.1)) determines a function $v_{N+1}$ that satisfies equation Q1(0) (2.5). $v_{N}$ and $v_{N+1}$ obey the relation

$$
\left(\widetilde{v}_{N+1}-v_{N+1}\right)\left(\widetilde{v}_{N}-v_{N}\right)=a^{2} v_{N} \widetilde{v}_{N}, \quad\left(\widehat{v}_{N+1}-v_{N+1}\right)\left(\widehat{v}_{N}-v_{N}\right)=b^{2} v_{N} \widehat{v}_{N},
$$

which is an auto BT of Q1(0).

Proof. The first part of the theorem holds due to Lemma 3.2. For the second part, since $\left(v_{N}, V_{N}\right)$ is a solution pair of the BT (3.1), they have compatibility $\widehat{\widetilde{A}}=\widetilde{\widehat{A}}$, and so does $V_{N+1}$. Then, on the basis of Lemma 3.1, $v_{N+1}$ defined by (3.3b) solves Q1(0). For the relation (3.4), substituting (3.3a) into (3.3b) and making use of (3.1) with $(v, V)=\left(v_{N}, V_{N}\right)$, we arrive at (3.4), which provides an auto BT for Q1(0). In fact, there is a non-auto BT [4]

$$
(u-\widetilde{u})(v-\widetilde{v})=p\left(v \widetilde{v}-\delta^{2}\right), \quad(u-\widehat{u})(v-\widehat{v})=q\left(v \widehat{v}-\delta^{2}\right),
$$

to map $v$ to $u$ from $\mathrm{Q} 1(0)$ to $\mathrm{Q} 1(\delta)$. It holds as well for the degenerated case $\delta=0$, in which both $v$ and $u$ are solutions of $\mathrm{Q} 1(0)$.

\subsection{Rational solutions of Q1(0) and lpmKdV}

Theorem 3.3 describes an iterative mechanism to generate new solutions for Q1(0) and the lpmKdV equation. Thus, if we start from a simple solution pair, e.g., $\left(v_{1}=a n+b m+\gamma_{1}, V_{1}=1\right)$, we can generate a sequence of rational solutions to Q1(0) and the lpmKdV equation. Some low order solutions in this sequence are

$$
\begin{aligned}
v_{1}= & x_{1}, \quad V_{1}=1, \\
v_{2}= & \frac{1}{3}\left(x_{1}^{3}-x_{3}\right), \quad V_{2}=x_{1}, \\
v_{3}= & \frac{1}{x_{1}}\left(\frac{1}{45} x_{1}^{6}-\frac{1}{9} x_{1}^{3} x_{3}+\frac{1}{5} x_{1} x_{5}-\frac{1}{9} x_{3}^{2}\right), \quad V_{3}=\frac{x_{1}^{3}-x_{3}}{3 x_{1}}, \\
v_{4}= & \frac{3}{x_{1}^{3}-x_{3}}\left(\frac{1}{4725} x_{1}^{10}-\frac{1}{315} x_{1}^{7} x_{3}+\frac{1}{75} x_{1}^{5} x_{5}-\frac{1}{27} x_{1} x_{3}^{3}\right. \\
& \left.-\frac{1}{25} x_{5}^{2}+\frac{1}{15} x_{1}^{2} x_{3} x_{5}-\frac{1}{21} x_{1}^{3} x_{7}+\frac{1}{21} x_{3} x_{7}\right), \\
V_{4}= & \frac{3}{x_{1}^{3}-x_{3}}\left(\frac{1}{45} x_{1}^{6}-\frac{1}{9} x_{1}^{3} x_{3}+\frac{1}{5} x_{1} x_{5}-\frac{1}{9} x_{3}^{2}\right),
\end{aligned}
$$

where

$$
x_{i}=a^{i} n+b^{i} m+\gamma_{i}, \quad \gamma_{i} \in \mathbb{C}, \quad i=1,2, \ldots
$$

We note that $\left\{V_{N}\right\}$ are different from the rational solutions of the lpmKdV equation obtained in [17] as a reduction of the Hirota-Miwa equation.

In the following we prove that if we start from (3.6), all the solutions generated from (3.3) are meaningful. First, let us look at non-zero property. 
Lemma 3.4. Suppose $a>0, b>0, v_{N}(0,0)>0$, and we restrict $(n, m)$ in the first quadrant $\{n \geq 0, m \geq 0\}$. Then $v_{N}$ and $V_{N}$ generated from (3.3) with (3.6a) satisfy $v_{N}>0, V_{N}>0$.

Proof. Obviously, under assumption of the lemma, from (3.6a) we have $v_{1}>0, V_{1}>0$ and $V_{2}=v_{1} / V_{1}>0$. Then, suppose that $v_{N}>0, V_{N}>0$ and consequently $V_{N+1}=v_{N} / V_{N}>0$. Next, from (3.3b) we have

$$
v_{N+1}(n+1, m)-v_{N+1}(n, m)>0, \quad v_{N+1}(n, m+1)-v_{N+1}(n+1, m)>0 .
$$

This implies

$$
v_{N+1}(n, m)>v_{N+1}(n-1, m)>v_{N+1}(n-1, m-1)>\cdots>v_{N+1}(0,0) .
$$

If we take "integration" constant $v_{N+1}(0,0)>0$, then $v_{N+1}(n, m)$ must be positive in quadrant $\{n \geq 0, m \geq 0\}$.

Next, we observe that in $v_{1}, v_{2}$ and $v_{3}$ the order of leading terms (in terms of $x_{1}$ ) are respectively 1,3 and 5 . Now we prove all the $v_{N}$ defined through (3.3) with (3.6a) are distinct in the sense of having different leading orders in terms of $x_{1}$.

Lemma 3.5. $v_{N}$ has a leading order $2 N-1$ in terms of $x_{1}$ and $V_{N}$ has a leading order $N-1$ in the same sense.

Proof. From (3.6) we can suppose the lemma is correct up to some integer $N$. Then one can find

$$
V_{N+1}=\frac{v_{N}}{V_{N}} \sim O\left(x_{1}^{N}\right),
$$

and from $(3.3 b)$

$$
\widetilde{v}_{N+1}-v_{N+1}=a V_{N} \widetilde{V}_{N} \sim O\left(x_{1}^{2 N}\right), \quad \widehat{v}_{N+1}-v_{N+1}=b V_{N} \widehat{V}_{N} \sim O\left(x_{1}^{2 N}\right),
$$

which means $v_{N+1} \sim O\left(x_{1}^{2 N+1}\right)$. Based on mathematical induction, the lemma holds.

We conclude the following.

Theorem 3.6. The iteration relation (3.3) is meaningful in terms of generating distinct rational solutions from initial solutions (3.6a) for $\mathrm{Q} 1(0)$ and the $\mathrm{lpmKdV}$ equation. These solutions are positive at least on the first quadrant $\{n \geq 0, m \geq 0\}$ if we take $a>0, b>0, v_{N}(0,0)>0$.

Note that not all solutions can be effectively iterated through (3.3). For example,

$$
v_{1}=\alpha^{n} \beta^{m}, \quad V_{1}=v_{1}^{\frac{1}{2}}
$$

are confined in (3.3) due to $V_{2}=v_{1} / V_{1}=V_{1}$. Here the parameterizations for $a$ and $b$ are $a^{2}=p=(1-\alpha)^{2} / \alpha, b^{2}=q=(1-\beta)^{2} / \beta$.

\subsection{Solutions to $\mathrm{Q} 1(\delta)$}

The iteration (3.3) can be extended to $N \leq 0$.

Lemma 3.7. Define

$$
v_{-N}=-\frac{1}{v_{N+1}}, \quad V_{-N}=(-1)^{N+1} \frac{1}{V_{N+2}}, \quad \text { for } \quad N \geq 0 .
$$

Then the iteration relation (3.3) can be extended to $N \in \mathbb{Z}$. 
This lemma can be checked directly.

Note that the extension does not lead to new solutions to Q1(0) and the lpmKdV equation because these two equations are invariant under transformations of type $u \rightarrow c / u$. However, the extension does bring more rational solutions to $\mathrm{Q} 1(\delta)$.

Theorem 3.8. For the pair $\left(v_{N}, V_{N}\right)$ determined by (3.3) with $N \in \mathbb{Z}$, function

$$
u_{N}=v_{N}+\frac{\delta^{2}}{v_{N-2}}, \quad N \in \mathbb{Z},
$$

gives a sequence of solutions to $\mathrm{Q} 1(\delta) . u_{N}$ and $v_{N-1}$ are connected via

$$
\widetilde{u}_{N}-u_{N}=\frac{a^{2}\left(v_{N-1} \widetilde{v}_{N-1}-\delta^{2}\right)}{\widetilde{v}_{N-1}-v_{N-1}}, \quad \widehat{u}_{N}-u_{N}=\frac{b^{2}\left(v_{N-1} \widehat{v}_{N-1}-\delta^{2}\right)}{\widehat{v}_{N-1}-v_{N-1}},
$$

which is the non-auto BT (3.5) between $\mathrm{Q} 1(\delta)$ and $\mathrm{Q} 1(0)$. Also, (3.9) agrees with the chain

$$
u_{1} \stackrel{\delta=0}{\longrightarrow} v_{1} \stackrel{\mathrm{BT}(3.10)}{\longrightarrow} u_{2} \stackrel{\delta=0}{\longrightarrow} v_{2} \stackrel{\mathrm{BT}(3.10)}{\longrightarrow} u_{3} \ldots \ldots .
$$

Proof. We only need to prove $u_{N}$ defined by (3.9) satisfies (3.10). Since $\left\{v_{N}\right\}$ obey the BT (3.4), using which we can find

$$
\widetilde{v}_{k}-v_{k}=\frac{a^{2} \widetilde{v}_{k-1} v_{k-1}}{\widetilde{v}_{k-1}-v_{k-1}}, \quad \frac{1}{\widetilde{v}_{k-2}}-\frac{1}{v_{k-2}}=\frac{-a^{2}}{\widetilde{v}_{k-1}-v_{k-1}} .
$$

Then, from (3.9) by direct calculation we immediately have

$$
\widetilde{u}_{N}-u_{N}=\widetilde{v}_{N}-v_{N}+\delta^{2}\left(\frac{1}{\widetilde{v}_{N-2}}-\frac{1}{v_{N-2}}\right)=\frac{a^{2}\left(v_{N-1} \widetilde{v}_{N-1}-\delta^{2}\right)}{\widetilde{v}_{N-1}-v_{N-1}}
$$

which coincides with the first equation in (3.10). Similarly we can find $\widehat{u}_{N}-u_{N}$ satisfies the second equation in (3.10) as well.

Formula (3.9) provides an explicit relation between solutions of Q1 $(\delta)$ and Q1(0), where $\left\{v_{N}\right\}$ is a sequence generated from (3.3). For $v_{N}$ given as in (3.6), some rational solutions of Q1 $(\delta)$ generated from (3.9) are

$$
\begin{aligned}
& u_{1}=x_{1}-\frac{1}{3} \delta^{2}\left(x_{1}^{3}-x_{3}\right), \\
& u_{2}=\frac{1}{3}\left(x_{1}^{3}-x_{3}\right)-\delta^{2} x_{1}, \\
& u_{3}=\frac{1}{x_{1}}\left(\frac{1}{45} x_{1}^{6}-\frac{1}{9} x_{1}^{3} x_{3}+\frac{1}{5} x_{1} x_{5}-\frac{1}{9} x_{3}^{2}+\delta^{2}\right),
\end{aligned}
$$

where we have made use of relation (3.8) in order to get $v_{-1}$ and $v_{0}$.

Here we give two remarks.

Remark 3.9. There are some overlaps (dual forms) in the chain (3.9). Note that Q1 $(\delta)$ equation is formally invariant under transformation first replacing $u$ with $\varepsilon \delta^{2} u$ and then $\delta$ with $1 / \delta$ where $\varepsilon= \pm 1$, by which (3.9) is transformed into its dual form

$$
u_{N}=\varepsilon\left(\delta^{2} v_{N}+\frac{1}{v_{N-2}}\right),
$$

which gives a sequence of solutions to $\mathrm{Q} 1(\delta)$ as well. By the relation (3.8) given in Lemma 3.7, $u_{N}$ and $u_{3-N}$ in (3.9) are dual forms of each other. 
Remark 3.10. Not all the rational solutions of $\mathrm{Q} 1(\delta)$ are included in the chain $(3.9)$. We give two exceptions. One is

$$
u=\alpha n+\beta m+\gamma,
$$

where $\gamma$ is a constant and $\alpha, \beta$ are defined by parametrization

$$
p=\frac{c_{0}}{a^{2}-\delta^{2}}, \quad q=\frac{c_{0}}{b^{2}-\delta^{2}}, \quad \alpha=p a, \quad \beta=q b,
$$

with arbitrary constant $c_{0}$, and the other is

$$
u=\delta x_{1}^{2}+\delta \gamma_{0},
$$

where $x_{1}$ is defined as (3.7), $p, q$ are parameterized as in (3.2) and $\gamma_{0}$ is a constant.

\subsection{Rational solutions to Q2}

We make use of a non-auto BT between $\mathrm{Q} 1(\delta)(2.6)$ and Q2 (2.8) to derive rational solutions of Q2. The BT reads [4]

$$
\begin{aligned}
& \delta(u-\widetilde{u})(w-\widetilde{w})=p\left(2 u \widetilde{u}-\delta^{2} w-\delta^{2} \widetilde{w}\right)+\delta p^{2}(u+\widetilde{u}+\delta p), \\
& \delta(u-\widehat{u})(w-\widehat{w})=q\left(2 u \widehat{u}-\delta^{2} w-\delta^{2} \widehat{w}\right)+\delta q^{2}(u+\widehat{u}+\delta q) .
\end{aligned}
$$

When $u$ is given by (3.13a) with parametrization (3.13b), from (3.15) we can find

$$
w=\frac{u^{2}}{\delta^{2}}-\frac{c_{0} u}{\delta^{3}}+\frac{c_{0}^{2}}{2 \delta^{4}}+\left(\frac{a-\delta}{a+\delta}\right)^{n}\left(\frac{b-\delta}{b+\delta}\right)^{m} \gamma_{0}, \quad u=\alpha n+\beta m+\gamma,
$$

where $\gamma_{0}$ is a constant. This is not a pure rational solution.

For $u$ defined in (3.14) with parametrization (3.2), from (3.15) we find

$$
w=\frac{1}{5} x_{1}^{4}+\frac{2}{3} \gamma_{0} x_{1}^{2}+\frac{4 \gamma_{0} x_{3}}{3 x_{1}}+\frac{4 x_{5}}{5 x_{1}}+\gamma_{0}^{2} .
$$

For $u=u_{2}$ given by (3.12) with parametrization (3.2), from (3.15) we find

$$
\begin{aligned}
w= & \frac{1}{45 \delta\left(x_{1}-\delta\right)}\left[30 \delta^{3}\left(x_{1}^{3}-x_{3}\right)-15 \delta^{2}\left(x_{1}^{4}+2 x_{1} x_{3}\right)-3 \delta\left(x_{1}^{5}-10 x_{1}^{2} x_{3}-6 x_{5}\right)\right. \\
& \left.+2 x_{1}^{6}+18 x_{1} x_{5}-10 x_{3}^{2}-10 x_{1}^{3} x_{3}\right] .
\end{aligned}
$$

\subsection{Solutions to $\mathrm{H} 3(\delta)$}

To obtain solutions to $\mathrm{H} 3(\delta)$ we make use of $\mathrm{A} 1(\delta)$ (2.7). Solutions to $\mathrm{A} 1(\delta)$ can be obtained from those of $\mathrm{Q} 1(\delta)$ (2.6) through transformation [2]

$$
z=(-1)^{n+m} u \text {. }
$$

Similar to the triplet composed by (2.5), (2.3) and (3.1), A1 $(\delta)(2.7)$ with parametrization (3.2), $\mathrm{H} 3(\delta)(2.4)$ and their non-auto BT [4]

$$
\widetilde{z}+z-\delta a^{2}=a Z \widetilde{Z}, \quad \widehat{z}+z-\delta b^{2}=b Z \widehat{Z}
$$

constitute a consistent triplet, i.e., compatibility $\widehat{\widetilde{z}}=\widetilde{\widehat{z}}$ in (3.16) requires $Z$ satisfies (2.4) and $\widehat{\widetilde{Z}}=\widetilde{\widehat{Z}}$ requires $z$ satisfies $(2.7)$. Such a consistency leads to 


\section{Lemma 3.11.}

(1) Starting from any solution $z$ of (2.7), by integration through (3.16), the resulted $Z$ solves (2.4), and vice versa.

(2) any solution pair $(z, Z)$ of (3.16) gives a solution $z$ to (2.7) and $Z$ to (2.4).

Solution sequences of $\mathrm{A} 1(\delta)$ and $\mathrm{H} 3(\delta)$ are then given as follows.

Theorem 3.12. For $v_{N}$ and $V_{N}$ constructed in Theorem 3.3 and Lemma 3.7, function

$$
z_{N}=(-1)^{n+m}\left(v_{N}+\frac{\delta^{2}}{v_{N-2}}\right), \quad N \in \mathbb{Z},
$$

solves $\mathrm{A} 1(\delta)(2.7)$, and

$$
Z_{N}=(-1)^{\frac{n+m}{2}+\frac{1}{4}}\left(V_{N}+\frac{(-1)^{n+m} \delta}{V_{N-1}}\right), \quad N \in \mathbb{Z},
$$

solves $\mathrm{H} 3(\delta)(2.4)$.

Proof. Since $u_{N}$ defined in (3.9) solves Q1 $(\delta)$, it is obvious that (3.17) provides a solution to $\mathrm{A} 1(\delta)$. Besides, making use of iterative relation (3.3), one can find (3.17) and (3.18) provide a solution pair to (3.16), which proves the present theorem.

Here we list some solutions for $\mathrm{H} 3(\delta)$,

$$
\begin{aligned}
& Z_{1}=(-1)^{\frac{n+m}{2}+\frac{1}{4}}\left(1-(-1)^{n+m} \delta x_{1}\right), \\
& Z_{2}=(-1)^{\frac{n+m}{2}+\frac{1}{4}}\left(x_{1}+(-1)^{n+m} \delta\right), \\
& Z_{3}=(-1)^{\frac{n+m}{2}+\frac{1}{4}} \frac{x_{1}^{3}-x_{3}+3(-1)^{n+m} \delta}{3 x_{1}} .
\end{aligned}
$$

Similar to Q1 $(\delta)$, there are also overlaps (dual forms) in the chain (3.18) for H3 $(\delta)$. H3 $(\delta)$ is formally invariant under transformation first $Z \rightarrow \varepsilon \delta^{-1}(-1)^{n+m} Z$ and then $\delta \rightarrow-\delta^{-1}$ with $\varepsilon= \pm 1$, by which (3.18) becomes

$$
Z_{N}=\varepsilon(-1)^{\frac{n+m}{2}+\frac{1}{4}}\left(\delta(-1)^{n+m} V_{N}-\frac{1}{V_{N-1}}\right) .
$$

Thus, $Z_{N}$ and $Z_{3-N}$ in (3.18) are dual forms of each other in light of relation (3.8).

\section{Solutions of $\mathrm{H} 1$ and $\mathrm{H} 2$}

In this section we first derive solutions of $\mathrm{H} 1$ using a relation between $\mathrm{H} 1$ and the lpmKdV equation. Then from $\mathrm{H} 1$ we derive solutions of $\mathrm{H} 2$.

\section{$4.1 \quad H 1$}

There is a non-auto BT [13]

$$
\widetilde{u}-\widehat{u}=\frac{b \widetilde{V}-a \widehat{V}}{a b V}, \quad \widehat{\widetilde{u}}-u=\frac{b \widehat{\widetilde{V}}+a V}{a b \widehat{V}}
$$

to connect $\mathrm{H} 1(u)(2.1)$ and the $\operatorname{lpmKdV}(V)$ equation (2.3). We use it to derive solutions for $\mathrm{H} 1$ on the basis of the following fact. 
Lemma 4.1. When $V$ solves the lpmKdV equation (2.3), function u defined by (4.1) satisfies $\mathrm{H} 1$ (2.1) with parametrization

$$
p=-1 / a^{2}, \quad q=-1 / b^{2} .
$$

Proof. We rewrite the lpmKdV equation (2.3) as

$$
(b \widetilde{V}-a \widehat{V})(b \widehat{\widetilde{V}}+a V)=\left(b^{2}-a^{2}\right) V \widehat{V} .
$$

Then, multiplying both equations in (4.1) and making use of (4.3) we immediately reach H1 (2.1) provided $p, q$ are parameterized as (4.2).

From (4.1) we find

$$
\widetilde{\widetilde{u}}-u=\frac{V+\widetilde{\widetilde{V}}}{a \widetilde{V}}, \quad \widehat{\widehat{u}}-u=\frac{V+\widehat{\widehat{V}}}{b \widehat{V}},
$$

of which we make use to derive $u$ from known $V$.

For $V_{1}=1$ and $V_{2}=x_{1}$ given in (3.6), we derive same solution for $\mathrm{H} 1$,

$$
u_{1}=u_{2}=x_{-1}
$$

where $x_{-1}$ follows the definition (3.7) with $i=-1$. For $V_{3}$ and $V_{4}$ given in (3.6), we respectively find

$$
u_{3}=x_{-1}-\frac{1}{x_{1}}
$$

and

$$
u_{4}=x_{-1}-\frac{3 x_{1}^{2}}{x_{1}^{3}-x_{3}} .
$$

Note that the lpmKdV equation (2.3) is invariant under $V \rightarrow \frac{1}{V}$. So we can replace $V$ by $1 / V$ in (4.4) and get

$$
\widetilde{\widetilde{u}}-u=\frac{\widetilde{V}}{a}\left(\frac{1}{V}+\frac{1}{\widetilde{\widetilde{V}}}\right), \quad \widehat{\widehat{u}}-u=\frac{\widehat{V}}{b}\left(\frac{1}{V}+\frac{1}{\widehat{\widehat{V}}}\right) .
$$

One may wonder if the above relation can be used to generate more solutions for H1. However, making use of iterative relations (3.3) we find

$$
\widetilde{\widetilde{u}}_{N+1}-u_{N+1}=\frac{V_{N+1}+\widetilde{\widetilde{V}}_{N+1}}{a \widetilde{V}_{N+1}}=\frac{\widetilde{V}_{N}}{a}\left(\frac{1}{V_{N}}+\frac{1}{\widetilde{\widetilde{V}}_{N}}\right),
$$

and a same formula for $\left({ }^{\wedge}, b\right)$, which means $V \rightarrow \frac{1}{V}$ does not lead to new solutions for H1. This can also explain the fact $u_{1}=u_{2}$ due to $V_{1}=\frac{1}{V_{1}}=1$.

\section{$4.2 \quad \mathrm{H} 2$}

Again, H2 (2.2), H1 (2.1) and their non-auto BT [4]

$$
v+\widetilde{v}+p=2 u \widetilde{u}, \quad v+\widehat{v}+q=2 u \widehat{u}
$$

constitute a consistent triplet with parametrization (4.2). Then, from solutions (4.5) of H1 and $\mathrm{BT}$ (4.6), we find the following rational solutions for $\mathrm{H} 2$ :

$$
v_{1}=v_{2}=x_{-1}^{2}, \quad v_{3}=x_{-1}^{2}-\frac{2 x_{-1}}{x_{1}}, \quad v_{4}=x_{-1}^{2}-\frac{6 x_{1}\left(x_{1} x_{-1}-1\right)}{x_{1}^{3}-x_{3}} .
$$




\section{Rational solutions in determinant form}

From the previous section it is understood that the sequence $\left\{V_{N}\right\}$ plays a crucial role in constructing solutions in the whole paper. With regard to rational solutions, it is hard to do "integration" from (3.3b) to get high order $v_{N}$ and consequently it is difficult to get high order $V_{N}$. In this section we aim to construct Casoratian expressions for $v_{N}$ and $V_{N}$, as well as rational solutions of other equations.

\subsection{Bilinear relation of $V_{N}$ and $v_{N}$}

We express

$$
V_{N}=\frac{P_{N-1}}{P_{N-2}}
$$

and it then follows from (3.3a) that

$$
v_{N}=\frac{P_{N}}{P_{N-2}}
$$

From $V_{2}=x_{1}$ we introduce

$$
P_{0}=1, \quad P_{1}=x_{1},
$$

and from (3.6) we find successively

$$
\begin{aligned}
& P_{2}=\frac{x_{1}^{3}-x_{3}}{3} \\
& P_{3}=\frac{1}{45} x_{1}^{6}-\frac{1}{9} x_{1}^{3} x_{3}+\frac{1}{5} x_{1} x_{5}-\frac{1}{9} x_{3}^{2}, \\
& P_{4}=\frac{1}{4725} x_{1}^{10}-\frac{1}{315} x_{1}^{7} x_{3}+\frac{1}{75} x_{1}^{5} x_{5}-\frac{1}{27} x_{1} x_{3}^{3}-\frac{1}{25} x_{5}^{2}+\frac{1}{15} x_{1}^{2} x_{3} x_{5}-\frac{1}{21} x_{1}^{3} x_{7}+\frac{1}{21} x_{3} x_{7},
\end{aligned}
$$

where $P_{4}$ is obtained from the relation $\frac{P_{4}}{P_{3}}=V_{5}=\frac{v_{4}}{V_{4}}$.

Viewing (5.1) as transformations, the BT (3.3b) yields

$$
\widetilde{\bar{P}} \underline{P}-\bar{P} \underline{\widetilde{P}}=a P \widetilde{P}, \quad \widehat{\bar{P}} \underline{P}-\bar{P} \underline{\widehat{P}}=b P \widehat{P},
$$

where

$$
P \doteq P_{N}, \quad \bar{P} \doteq P_{N+1}, \quad \underline{P} \doteq P_{N-1} .
$$

This is a bilinear system for polynomials $\left\{P_{N}\right\}$. Note that based on (3.8) the relations (5.1) and (5.2) can be extended to $N \in \mathbb{Z}$ by defining

$$
P_{-N}=(-1)^{\left[\frac{N}{2}\right]} P_{N-1}
$$

where $[\cdot]$ denotes the greatest integer function.

In Section 5.3 we will give a Casoratian form of $P$. To achieve that, we make use of H1. 


\subsection{Casoratian form of rational solutions of $\mathrm{H1}$}

For H1 (2.1), using 3D consistency we have its BT

$$
(\widetilde{u}-\bar{u})(\widetilde{\bar{u}}-u)=a^{-2}-k^{-2}, \quad(\widehat{u}-\bar{u})(\widehat{\bar{u}}-u)=b^{-2}-k^{-2},
$$

where $\bar{u}$ stands for a new solution of H1, we adopt parametrization (4.2) and the arbitrary number $k^{-2}=r$ acts as a "soliton number" which leads to a new soliton (cf. [14]). Now we remove the term $k^{-2}$ from (5.4), i.e., taking $r=0$, and consequently we have

$$
(\widetilde{u}-\bar{u})(\widetilde{\bar{u}}-u)=a^{-2}, \quad(\widehat{u}-\bar{u})(\widehat{\bar{u}}-u)=b^{-2},
$$

which can generate a rational part in the new solution $\bar{u}$.

To find solutions from (5.5), first, we introduce

$$
\begin{aligned}
& \widetilde{u}-\bar{u}=\frac{f \widetilde{\bar{f}}}{a \widetilde{f} \bar{f}}, \\
& \widetilde{\bar{u}}-u=\frac{\widetilde{f f \bar{f}}}{a f \widetilde{\bar{f}}}, \\
& \widehat{u}-\bar{u}=\frac{f \widehat{\bar{f}}}{b \widehat{f f}}, \\
& \widehat{\bar{u}}-u=\frac{\widehat{f \widetilde{f}}}{b \hat{\bar{f}}},
\end{aligned}
$$

which provide a factorization of (5.5). Such an assumption coincides with the previous results. In fact, suppose $V=\bar{f} / f$, then from (5.6) we can find $\widetilde{u}-\widehat{u}$ and $\widehat{\widetilde{u}}-u$ agree with (4.1) and $\widetilde{\widetilde{u}}-u$ and $\widehat{\widehat{u}}-u$ agree with (4.4). Then we introduce

$$
u=x_{-1}-\frac{g}{f}, \quad \bar{u}=x_{-1}-\frac{\bar{g}}{\bar{f}},
$$

by which we bilinearize (5.6) as

$$
\begin{aligned}
& \bar{g} \widetilde{f}-\bar{f} \widetilde{g}+\frac{1}{a}(\bar{f} \tilde{f}-\widetilde{\bar{f}} f)=0, \\
& g \widetilde{\bar{f}}-f \widetilde{\bar{g}}-\frac{1}{a}(\bar{f} \widetilde{f}-\widetilde{\bar{f}} f)=0, \\
& \bar{g} \widehat{f}-\bar{f} \widehat{g}+\frac{1}{b}(\bar{f} \widehat{f}-\widehat{\bar{f}} f)=0, \\
& g \widehat{\bar{f}}-f \hat{\bar{g}}-\frac{1}{b}(\bar{f} \widehat{f}-\widehat{\bar{f}} f)=0 .
\end{aligned}
$$

Next, we introduce Casoratian forms for $f, \bar{f}, g$ and $\bar{g}$. Consider function

$$
\begin{aligned}
& \psi_{i}(n, m, l)=\psi_{i}^{+}(n, m, l)+\psi_{i}^{-}(n, m, l), \\
& \psi_{i}^{ \pm}(n, m, l)=\varrho_{i}^{ \pm}\left(1 \pm s_{i}\right)^{l}\left(1 \pm a s_{i}\right)^{n}\left(1 \pm b s_{i}\right)^{m},
\end{aligned}
$$

where $\varrho_{i}^{ \pm}$and $s_{i}$ are nonzero constants ${ }^{1}$. This can be used to construct soliton solutions for H1 equation $(\text { cf. [14] })^{2}$. To derive the rational solutions obtained in the previous section, we take

$$
\varrho_{i}^{ \pm}= \pm \frac{1}{2} \exp \left[-\sum_{j=1}^{\infty} \frac{\left(\mp s_{i}\right)^{j}}{j} \gamma_{j}\right]
$$

\footnotetext{
${ }^{1}$ If $\varrho_{i}^{ \pm}$are independent on $s_{i}$, in practice in $(5.9)$ we replace $\left(1 \pm s_{i}\right)^{l}$ with $\left(1 \pm s_{i}\right)^{l+l_{0}}$ and suppose $l_{0}$ is either a large enough integer or a non-integer so that the derivative $\left.\partial_{s_{i}}^{h}\left(1 \pm s_{i}\right)^{l+l_{0}}\right|_{s_{i}=0} \neq 0$.

${ }^{2}$ One needs to use gauge property of bilinear $\mathrm{H} 1$ and make certain extension from $\left( \pm s_{i}\right)^{l+l_{0}}$ to $\left(1 \pm s_{i}\right)^{l+l_{0}}$.
} 
with arbitrary constant $\gamma_{j}$. Then we expand $\psi_{i}^{ \pm}(n, m, l)$ as

$$
\psi_{i}^{ \pm}(n, m, l)= \pm \frac{1}{2} \sum_{h=0}^{\infty} \alpha_{h}^{ \pm} s_{i}^{h}, \quad \alpha_{h}^{ \pm}= \pm\left.\frac{2}{h !} \partial_{s_{i}}^{h} \psi_{i}^{ \pm}\right|_{s_{i}=0} .
$$

By noticing that

$$
\psi_{i}^{ \pm}(n, m, l)= \pm \frac{1}{2} \exp \left[-\sum_{j=1}^{\infty} \frac{\left(\mp s_{i}\right)^{j}}{j} \stackrel{\circ}{x}_{j}\right], \quad \stackrel{\circ}{x}_{j}=x_{j}+l,
$$

where $x_{j}$ are exactly defined as (3.7), all $\left\{\alpha_{h}^{ \pm}\right\}$can be expressed in terms of $\left\{x_{j}\right\}$. For $\left\{\alpha_{h}^{+}\right\}$we have

$$
\alpha_{h}^{ \pm} \doteq \alpha_{h}^{ \pm}(n, m, l)=(\mp 1)^{h} \sum_{\|\mu\|=h}(-1)^{|\mu|} \frac{\stackrel{\circ}{x}^{\mu}}{\mu !},
$$

where

$$
\begin{aligned}
& \mu=\left(\mu_{1}, \mu_{2}, \ldots\right), \quad \mu_{j} \in\{0,1,2, \ldots\}, \quad\|\mu\|=\sum_{j=1}^{\infty} j \mu_{j}, \\
& |\mu|=\sum_{j=1}^{\infty} \mu_{j}, \quad \mu !=\mu_{1} ! \cdot \mu_{2} ! \cdots, \quad \stackrel{\circ}{\mathbf{x}}^{\mu}=\left(\frac{\stackrel{\circ}{x}_{1}}{1}\right)^{\mu_{1}}\left(\frac{\stackrel{\circ}{2}_{2}}{2}\right)^{\mu_{2}} \cdots .
\end{aligned}
$$

The first few $\alpha_{h}^{+}$are

$$
\begin{aligned}
& \alpha_{0}^{+}=1, \quad \alpha_{1}^{+}=\stackrel{\circ}{x}_{1}, \quad \alpha_{2}^{+}=\frac{1}{2}\left(\stackrel{\circ}{x}_{1}^{2}-\stackrel{\circ}{x}_{2}\right), \quad \alpha_{3}^{+}=\frac{1}{6}\left(\stackrel{\circ}{x}_{1}^{3}-3 \stackrel{\circ}{x}_{1} \stackrel{\circ}{x}_{2}+2 \stackrel{\circ}{x}_{3}\right), \\
& \alpha_{4}^{+}=\frac{1}{24}\left(\stackrel{\circ}{x}_{1}^{4}-6 \stackrel{\circ}{x}_{1}^{2} \stackrel{\circ}{x}_{2}+8 \stackrel{\circ}{x}_{1} \stackrel{\circ}{x}_{3}+3 \stackrel{\circ}{x}_{2}^{2}-6 \stackrel{\circ}{x}_{4}\right), \\
& \alpha_{5}^{+}=\frac{1}{120}\left(\stackrel{\circ}{x}_{1}^{5}-10 \stackrel{\circ}{x}_{2} \stackrel{\circ}{x}_{1}^{3}+20 \stackrel{\circ}{x}_{3} \stackrel{\circ}{x}_{1}^{2}+15 \stackrel{\circ}{x}_{2}^{2} \stackrel{\circ}{x}_{1}-30 \stackrel{\circ}{x}_{4} \stackrel{\circ}{x}_{1}-20 \stackrel{\circ}{x}_{2} \stackrel{\circ}{x}_{3}+24 \stackrel{\circ}{x}_{5}\right) .
\end{aligned}
$$

Introduce a column vector

$$
\alpha(n, m, l)=\left(\alpha_{0}, \alpha_{1}, \ldots, \alpha_{N-1}\right)^{\mathrm{T}}, \quad \alpha_{j}=\alpha_{2 j+1}^{+} .
$$

With $\alpha(n, m, l)$ as a basic column vector we introduce Casoratians w.r.t. shifts in $l$ :

$$
\begin{aligned}
& f=|\widehat{N-1}|_{\mathrm{R}}=|\alpha(n, m, 0), \alpha(n, m, 1), \ldots, \alpha(n, m, N-1)|, \\
& \bar{f}=|\widehat{N}|_{\mathrm{R}}, \quad g=|\widehat{N-2}, N|_{\mathrm{R}}-N f, \quad \bar{g}=|\widehat{N-1}, N+1|_{\mathrm{R}}-(N+1) \bar{f} .
\end{aligned}
$$

Some $f, g$ of low orders are

$$
\begin{aligned}
& f_{N=1}=x_{1}, \quad g_{N=1}=1, \\
& f_{N=2}=\frac{x_{1}^{3}-x_{3}}{3}, \quad g_{N=2}=x_{1}^{2}, \\
& f_{N=3}=\frac{1}{45} x_{1}^{6}-\frac{1}{9} x_{1}^{3} x_{3}+\frac{1}{5} x_{1} x_{5}-\frac{1}{9} x_{3}^{2}, \quad g_{N=3}=\frac{2}{15} x_{1}^{5}-\frac{1}{3} x_{1}^{2} x_{3}+\frac{1}{5} x_{5} .
\end{aligned}
$$

Through (5.7), $(f, g)$ with $N=1,2$ provide solutions (4.5b) and (4.5c) for H1. For general $N$, we have the following. 
Theorem 5.1. The Casoratians (5.14) solve the bilinear BT (5.8) and (5.7) provides rational solutions to $\mathrm{H} 1$.

Proof will be given in Appendix A.

Remark 5.2. There is an alternative choice for the Casoratians (5.14), which are given by just replacing the basic column vector $\alpha$ given in (5.13) by

$$
\beta(n, m, l)=\left(\beta_{0}, \beta_{1}, \ldots, \beta_{N-1}\right)^{\mathrm{T}}, \quad \beta_{j}=\alpha_{2 j}^{+},
$$

where $\alpha_{2 j}^{+}$are defined in (5.12), or equivalently,

$$
\beta_{j}=\left.\frac{1}{(2 j) !} \partial_{s_{i}}^{2 j} \psi_{i}\right|_{s_{i}=0}
$$

with

$$
\varrho_{i}^{ \pm}=\frac{1}{2} \exp \left[-\sum_{j=1}^{\infty} \frac{\left(\mp s_{i}\right)^{j}}{j} \gamma_{j}\right] .
$$

\subsection{Casoratian solutions to (5.2)}

We can make use of the BT of $\mathrm{H} 1$ to obtain solutions to bilinear equation (5.2). By the compatibility of (5.6a) and (5.6b), i.e., $\left(E_{n}-E_{N}\right)\left(E_{n} E_{N}-1\right) u=\left(E_{n} E_{N}-1\right)\left(E_{n}-E_{N}\right) u$ where $E_{N} f=\bar{f}$, we find

$$
\frac{\widetilde{\bar{f}} f-\overline{\bar{f}} \widetilde{f}}{\widetilde{\bar{f}} \bar{f}}=E_{n}\left(\frac{\widetilde{\bar{f}} f-\overline{\bar{f}} \tilde{f}}{\widetilde{\bar{f}} \bar{f}}\right) .
$$

Similarly,

$$
\frac{\widehat{\bar{f}} f-\overline{\bar{f}} \widehat{f}}{\widehat{\bar{f}} \bar{f}}=E_{m}\left(\frac{\widehat{\overline{\bar{f}}} f-\overline{\bar{f}} \hat{f}}{\widehat{\bar{f}} \bar{f}}\right) .
$$

This means

$$
\widetilde{\bar{f}} f-\overline{\bar{f}} \widetilde{f}=\lambda_{1}(m, N) \stackrel{\widetilde{f}}{f}, \quad \widehat{\bar{f}} f-\overline{\bar{f}} \widehat{f}=\lambda_{2}(n, N) \widehat{\bar{f}} \bar{f} .
$$

Next we go to prove $\lambda_{1}(m, N)=a$ and $\lambda_{2}(n, N)=b$. Again, from (5.6), we can derive

$$
\overline{\bar{u}}-u=\frac{\bar{f} \tilde{f}}{a \widetilde{\bar{f}} f}-\frac{\bar{f} \widetilde{\bar{f}}}{a \widetilde{\bar{f}} \overline{\bar{f}}}=\frac{\bar{f} \hat{f}}{b \widehat{\bar{f} f}}-\frac{\bar{f} \hat{\bar{f}}}{b \widehat{\bar{f}} \overline{\bar{f}}} .
$$

Using (5.17) to eliminate $\widetilde{\bar{f}}$ and $\widehat{\overline{\bar{f}}}$ from the above equation, we find

$$
\overline{\bar{u}}-u=-\lambda_{1}(m, N) \frac{\bar{f}^{2}}{a f \overline{\bar{f}}}=-\lambda_{2}(n, N) \frac{\bar{f}^{2}}{b f \overline{\bar{f}}},
$$

which means

$$
a \lambda_{2}(n, N)=b \lambda_{1}(m, N),
$$


and it then follows that both $\lambda_{1}$ and $\lambda_{2}$ must be $(n, m)$-independent. We assume

$$
\gamma(N)=\lambda_{1} / a=\lambda_{2} / b,
$$

and then (5.17) yields

$$
\gamma(N)=\frac{\widetilde{\bar{f}} f-\overline{\bar{f}} \tilde{f}}{a \widetilde{\widetilde{f}} \bar{f}}=\frac{\widehat{\overline{\bar{f}}} f-\overline{\bar{f}} \widehat{f}}{b \widehat{\bar{f}} \bar{f}} .
$$

To determine the value of $\gamma(N)$, we investigate properties of $f$ near the point $(n, m)=(0,0)$, which are presented through the following lemmas.

Lemma 5.3. According to the definitions of $u$ in (5.7), $f$ and $g$ in (5.14) and $\alpha_{h}^{ \pm}$in (5.12), we find the value of $\left.\alpha_{h}^{ \pm}\right|_{(n, m)=(0,0)}$ is independent of $(a, b)$, and so are $f(0,0), g(0,0)$ and $u(0,0)$. Then, from (5.18) we find that $\gamma(N)$ must be independent of $(a, b)$.

Lemma 5.4. For same $N$, there exists relation

$$
f_{N}(\alpha(n, m, l))=f_{N+1}(\beta(n, m, l)),
$$

where $\alpha(n, m, l)$ and $\beta(n, m, l)$ are respectively $N$-th order and $(N+1)$-th order column vectors defined as (5.13) and (5.16). Here and below $f_{N}(\psi)$ stands for a $N$-th order Casoratian $|\widehat{N-1}|$ composed by a $N$-th order basic column vector $\psi$.

Proof. First, noticing that relation

$$
\psi_{i}^{ \pm}(n, m, l+1)-\psi_{i}^{ \pm}(n, m, l)= \pm s_{i} \psi_{i}^{ \pm}(n, m, l),
$$

from the definition of $\alpha_{h}^{+}$in (5.11), we immediately get

$$
\alpha_{h}^{+}(n, m, l+1)-\alpha_{h}^{+}(n, m, l)=\alpha_{h-1}^{+}(n, m, l), \quad h \geq 1,
$$

from which, taking $h=2 j$, we reach

$$
\beta_{j}(n, m, l+1)-\beta_{j}(n, m, l)=\alpha_{j-1}(n, m, l), \quad j \geq 1 .
$$

It then follows that

$$
\beta(n, m, l+1)-\beta(n, m, l)=\left(\begin{array}{c}
0 \\
\alpha(n, m, l)
\end{array}\right),
$$

where $\alpha(n, m, l)$ and $\beta(n, m, l)$ are respectively $N$-th order and $(N+1)$-th order column vectors defined as (5.13) and (5.16). This immediately leads to the relation (5.20).

Lemma 5.5. For Casoratian $f_{N}(\alpha(n, m, l))$, the relation

$$
f_{N}(\alpha(1,0, l))=a^{N} f_{N-1}(\alpha(0,0, l))+O\left(a^{N-1}\right)
$$

holds.

Proof.

$$
\begin{aligned}
f_{N}(\alpha(1,0, l)) & =f_{N}(a \beta(0,0, l)+\alpha(0,0, l)) \\
& =a^{N} f_{N}(\beta(0,0, l))+O\left(a^{N-1}\right)=a^{N} f_{N-1}(\alpha(0,0, l))+O\left(a^{N-1}\right),
\end{aligned}
$$

where we have made use of relation (5.20). 
With this lemma, for $f=f_{N}(\alpha(n, m, l))$ in (5.19), we have

$$
\begin{aligned}
& \left.\widetilde{f}\right|_{n=m=0}=\left.a^{N} \underline{f}\right|_{n=m=0}+O\left(a^{N-1}\right),\left.\quad \widetilde{\bar{f}}\right|_{n=m=0}=\left.a^{N+1} f\right|_{n=m=0}+O\left(a^{N}\right), \\
& \left.\widetilde{\bar{f}}\right|_{n=m=0}=\left.a^{N+2} \bar{f}\right|_{n=m=0}+O\left(a^{N+1}\right) .
\end{aligned}
$$

Then, since $\gamma(N)$ is independent of $a$, from (5.19) we arrive at

$$
\gamma(N)=\left.\lim _{a \rightarrow \infty} \frac{\widetilde{\bar{f}} f-\overline{\bar{f}} \tilde{f}}{a \widetilde{\bar{f}} \bar{f}}\right|_{n=m=0}=1 .
$$

We can sum up this subsection with the following theorem.

Theorem 5.6. The Casoratian $f=f_{N}(\alpha(n, m, l))$ solves bilinear equation set

$$
\begin{aligned}
& \widetilde{\bar{f}} f-\overline{\bar{f}} \widetilde{f}=a \widetilde{\bar{f}} \bar{f}, \\
& \widehat{\bar{f}} f-\overline{\bar{f}} \widehat{f}=b \widehat{\bar{f}} \bar{f} .
\end{aligned}
$$

$P=f_{N}(\alpha(n, m, l))$ provides a Casoratian form of solution to (5.2). By defining

$$
f_{-N}=(-1)^{\left[\frac{N}{2}\right]} f_{N-1}, \quad f_{0}=1,
$$

one can consistently extend (5.22) to $N \in \mathbb{Z}$, which coincides with (5.3).

\subsection{Casoratian rational solutions to $\mathrm{H} 2$ and a sum-up}

We can derive Casoratian rational solutions for H2 through non-auto BT (4.6), in which we suppose

$$
u=x_{-1}-\frac{g}{f}, \quad v=x_{-1}^{2}-2\left(x_{-1}+N\right) \frac{g}{f}+\frac{h}{f}-N^{2} .
$$

Then BT (4.6) is bilinearized as

$$
\begin{aligned}
& f \widetilde{h}+\widetilde{f} h+2\left(a^{-1}-N\right) g \widetilde{f}-2\left(a^{-1}+N\right) \widetilde{g} f-2 g \widetilde{g}-2 N^{2} f \widetilde{f}=0, \\
& f \widehat{h}+\widehat{f} h+2\left(b^{-1}-N\right) g \widehat{f}-2\left(b^{-1}+N\right) \widehat{g} f-2 g \widehat{g}-2 N^{2} f \widehat{f}=0 .
\end{aligned}
$$

Based on the bilinear form we have

Theorem 5.7. The Casoratians

$$
\begin{aligned}
& f=|\widehat{N-1}|_{\mathrm{R}}, \quad g=|\widehat{N-2}, N|_{\mathrm{R}}-N f, \\
& h=|\widehat{N-2}, N+1|_{\mathrm{R}}+|\widehat{N-3}, N-1, N|_{\mathrm{R}}
\end{aligned}
$$

solve the bilinear BT system (5.25), in which the basic Casoratian column vector $\alpha$ is given by (5.13). Consequently, (5.24) provides rational solutions to $\mathrm{H} 1$ and $\mathrm{H} 2$.

Proof will be given in Appendix B.

Besides (5.15), some $h$ of low orders are

$$
\begin{aligned}
& h_{N=1}=x_{1}+2, \quad h_{N=2}=\frac{4}{3}\left(x_{1}^{3}-x_{3}\right)+4 x_{1}^{2}+2 x_{1}, \\
& h_{N=3}=\frac{1}{5} x_{1}^{6}-x_{1}^{3} x_{3}+\frac{9}{5} x_{1} x_{5}-x_{3}^{2}+\frac{4}{5} x_{1}^{5}-2 x_{1}^{2} x_{3}+\frac{6}{5} x_{5}+\frac{2}{3} x_{1}^{4}-\frac{2}{3} x_{1} x_{3},
\end{aligned}
$$

where all $\gamma_{i}=l_{i}$ in $x_{i}$. 
So far we have obtained Casoratian expressions for the rational solutions of Q1(0), lpmKdV, $\mathrm{Q} 1(\delta), \mathrm{H} 3(\delta), \mathrm{H} 1$ and $\mathrm{H} 2$. Noting that all these solutions are related to the rational solutions $V_{N}$ of the lpmKdV equation, it is necessary to express all these obtained solutions through the Casoratians with a unified $N$. We collect them in the following theorem.

Theorem 5.8. Suppose that

$$
\begin{aligned}
& f \doteq f_{N}=|\widehat{N-1}|_{\mathrm{R}}, \quad g \doteq g_{N}=|\widehat{N-2}, N|_{\mathrm{R}}-N f, \\
& h \doteq h_{N}=|\widehat{N-2}, N+1|_{\mathrm{R}}+|\widehat{N-3}, N-1, N|_{\mathrm{R}},
\end{aligned}
$$

and denote $\bar{f}=f_{N+1}$ and $\underline{f}=f_{N-1}$. Then the rational solutions for $\mathrm{Q} 1(0), \operatorname{lpmKdV}, \mathrm{Q} 1(\delta)$, $\mathrm{H} 3(\delta), \mathrm{H} 1$ and $\mathrm{H} 2$ are respectively

$$
\begin{aligned}
& \mathrm{Q} 1(0): \quad v_{N+2}=\frac{\overline{\bar{f}}}{f}, \\
& \operatorname{lpmKdV}: \quad V_{N+2}=\frac{\bar{f}}{f}, \\
& \mathrm{Q} 1(\delta): \quad u_{N+2}=\frac{\overline{\bar{f}}+\delta^{2} \underline{\underline{f}}}{f}, \\
& \mathrm{H} 3(\delta): \quad Z_{N+2}=(-1)^{\frac{n+m}{2}+\frac{1}{4}} \frac{\bar{f}+(-1)^{n+m} \delta \underline{f}}{f}, \\
& \text { H1: } \quad u_{N+2}=x_{-1}-\frac{g}{f}, \\
& \text { H2: } \quad v_{N+2}=x_{-1}^{2}-2\left(x_{-1}+N\right) \frac{g}{f}+\frac{h}{f}-N^{2} \text {. }
\end{aligned}
$$

\subsection{Rational solutions to Q2}

Now we come to the final equation, Q2. We start from the non-auto BT (3.15) in which we take parametrization (3.2) and $u$ to be $(5.28 \mathrm{c})$ which is a solution of Q1 $(\delta)$. Introduce auxiliary function

$$
w=y+\frac{u^{2}}{\delta^{2}}
$$

by which the BT (3.15) yields

$$
\begin{aligned}
& \widetilde{y}=\frac{\widetilde{u}-u-\delta p}{\widetilde{u}-u+\delta p} y+\frac{1}{\delta^{2}}(u+\delta p-\widetilde{u})(u+\delta p+\widetilde{u}), \\
& \widehat{y}=\frac{\widehat{u}-u-\delta q}{\widehat{u}-u+\delta q} y+\frac{1}{\delta^{2}}(u+\delta q-\widehat{u})(u+\delta q+\widehat{u}) .
\end{aligned}
$$

Then, making use of the relation (5.22), from (5.28c) we can find

$$
\begin{aligned}
& \widetilde{u}_{N+2}-u_{N+2}+\delta p=a \frac{(\bar{f}+\delta \underline{f})(\widetilde{\bar{f}}-\delta \tilde{f})}{f \widetilde{f}}, \\
& \widetilde{u}_{N+2}-u_{N+2}-\delta p=a \frac{(\bar{f}-\delta \underline{f})(\widetilde{\bar{f}}+\delta \widetilde{\widetilde{f}})}{f \widetilde{f}} .
\end{aligned}
$$

On the basis of the above relations together with their $\left(q,{ }^{\wedge}\right)$ version, and introducing

$$
\theta_{N+2}=y_{N+2} \frac{\bar{f}-\delta \underline{f f}}{\bar{f}+\delta \underline{f}},
$$


we then reduce (5.29) to

$$
\begin{aligned}
& \theta_{N+2}-\widetilde{\theta}_{N+2}=\frac{a(\bar{f}-\delta \underline{f})(\tilde{\bar{f}}-\delta \underline{\widetilde{f}})}{\delta^{2} f \widetilde{f}}\left(u_{N+2}+\widetilde{u}_{N+2}+\delta p\right), \\
& \theta_{N+2}-\widehat{\theta}_{N+2}=\frac{b(\bar{f}-\delta \underline{f})(\widehat{\bar{f}}-\delta \underline{\widehat{f}})}{\delta^{2} f \widehat{f}}\left(u_{N+2}+\widehat{u}_{N+2}+\delta q\right) .
\end{aligned}
$$

To solve this system we expand

$$
\theta_{N+2}=\sum_{i=-2}^{2} \theta_{N+2}^{(i)} \delta^{i}
$$

It then follows from (5.30) that

$$
\begin{aligned}
& \theta_{N+2}^{(-2)}-\widetilde{\theta}_{N+2}^{(-2)}=\frac{a \widetilde{\bar{f}} \bar{f}}{f^{2} \widetilde{\bar{f}}}(\widetilde{\bar{f}} f+\widetilde{f} \overline{\bar{f}}), \\
& \theta_{N+2}^{(-1)}-\widetilde{\theta}_{N+2}^{(-1)}=\frac{-2 a}{f^{2} \widetilde{f}^{2}}(\bar{f} \widetilde{\tilde{f}} \widetilde{\bar{f}} f+\widetilde{\bar{f}} \underline{f} \tilde{f} \overline{\bar{f}}), \\
& \theta_{N+2}^{(0)}-\widetilde{\theta}_{N+2}^{(0)}=\frac{a^{2}}{f^{2} \widetilde{f}^{2}}\left(\underline{\tilde{f}}^{2} \bar{f}^{2}-\underline{f}^{2} \underline{\bar{f}}^{2}\right)+\frac{2(\widetilde{\overline{\bar{f}}} \underline{\underline{\underline{f}}-\tilde{\widetilde{f}} \overline{\bar{f}})}}{f \widetilde{f}}, \\
& \theta_{N+2}^{(1)}-\widetilde{\theta}_{N+2}^{(1)}=\frac{-2 a}{f^{2} \widetilde{f}^{2}}(\underline{f} \underline{\widetilde{f}} \underline{\underline{\tilde{f}}} f+\widetilde{\widetilde{f}} \underline{\underline{f}} \tilde{f} \bar{f}), \\
& \left.\theta_{N+2}^{(2)}-\widetilde{\theta}_{N+2}^{(2)}=\frac{a \underline{\widetilde{f}}}{f^{2}} \frac{\tilde{\widetilde{f}^{2}}}{(\underline{\tilde{f}}} f+\widetilde{\tilde{f} f}\right),
\end{aligned}
$$

among which, except $\theta_{N+2}^{(0)}$, we find explicit expressions for $\theta_{N+2}^{(i)}$ in terms of $f$ :

$$
\begin{aligned}
& \theta_{N+2}^{(-1)}=-\frac{\overline{\bar{f}}^{2}}{f^{2}}, \quad \theta_{N+2}^{(-1)}=\frac{2 \overline{\bar{f}}^{2} \underline{f}+2 f^{2} \overline{\bar{f}}}{f^{2} \bar{f}}, \\
& \theta_{N+2}^{(1)}=-\frac{2{\underline{f^{2}}}^{2} \bar{f}+2 f^{2} \underline{\underline{f}}}{f^{2} \underline{f}}, \quad \theta_{N+2}^{(2)}=\frac{\underline{f}^{2}}{f^{2}} .
\end{aligned}
$$

For $\theta_{N+2}^{(0)}$ which is determined by (5.31c), the simplest two items are

$$
\theta_{2}^{(0)}=\frac{1}{3} x_{1}^{4}+\frac{2}{3} x_{1} x_{3}, \quad \theta_{3}^{(0)}=-\frac{1}{15} x_{1}^{4}+\frac{2}{3} x_{1} x_{3}+\frac{2 x_{5}}{5 x_{1}} .
$$

However, so far we do not find an explicit expression for $\theta_{N+2}^{(0)}$ in terms of $f$ and other auxiliary functions.

As a conclusion of rational solutions of Q2, we give the following theorem.

Theorem 5.9. Suppose that $f=f_{N}$ is defined as in (5.27). Our construction provides rational solutions of $\mathrm{Q} 2$ in the following form

$$
w_{N+2}=\frac{u_{N+2}^{2}}{\delta^{2}}+\frac{\bar{f}+\delta \underline{f}}{\bar{f}-\delta \underline{f}}\left(\frac{-\overline{\bar{f}}^{2}}{\delta^{2} f^{2}}+\frac{2 \overline{\bar{f}}^{2} \underline{f}+2 f^{2} \overline{\bar{f}}}{\delta f^{2} \bar{f}}+\theta_{N+2}^{(0)}-\frac{2 \delta \underline{\underline{f}}^{2} \bar{f}+2 \delta f^{2} \underline{\underline{f}}}{f^{2} \underline{f}}+\frac{\delta^{2} \underline{f}^{2}}{f^{2}}\right)
$$

where $u_{N+2}$ is given by $(5.28 \mathrm{c})$ and $\theta_{N+2}^{(0)}$ is determined by $(5.31 \mathrm{c})$.

We note that it might be not sufficient to call (5.33) a rational solution for arbitrary $N$, because for this moment we do not have a general solution form (like (5.32)) for $\theta_{N+2}^{(0)}$. 


\section{Conclusions}

In the paper we have derived rational solutions for the lpmKdV equation and some lattice equations in the ABS list. We make use of lpmKdV-Q1(0) consistent triplet to construct their rational solutions iteratively. This then becomes a starting point and through the route in

Fig. 1 to generate solutions for other equations. All these rational solutions are related to a unified $\tau$ function in Casoratian form, $f(\alpha)=|\widehat{N-1}|$, which obeys the bilinear superposition formula (5.22).

There are several interesting points we would like to remark. First, formula (3.9) reveals an explicit relation between certain solutions of $\mathrm{Q} 1(\delta)$ and Q1(0). This formula holds not only for rational solutions but also for solitons. Once we obtain $v_{N-2}$ and $v_{N}$ from (3.3), formula (3.9) gives a solution $u_{N}$ to $\mathrm{Q} 1(\delta)$, and these solutions provide a solution sequence for the chain (3.11) which is based on BT (3.5), i.e., (3.10). The second thing is about bilinear superposition formula (5.22) or (5.2). Casoratian $f$ with $\psi_{i}(5.9)$ as a basic entry is also a solution of bilinear equation

$$
(a+b) \underset{\sim}{\widehat{f}} \tilde{f}+(b-a) \underset{\sim}{f} \underset{\widetilde{f}}{\widehat{f}}=2 b f \widehat{f},
$$

as well as its dual version by switching $(a, \sim)$ and $(b, \widehat{ })$. (6.1) can be considered as a bilinear form of Hirota's discrete KdV equation (see [15] and [13, Section 8.4.1]). It was also derived from the Cauchy matrix approach as a bilinear form that is related to H1 (see [13, Section 9.4.3]). It is also well known that (6.1) can be derived as a reduction of the Hirota-Miwa equation, of which some rational solutions were derived from several different ways and reductions of few cases was already considered $[11,17]$. Here we can consider $(5.22)$ as a bilinear superposition formula of (6.1) for rational solutions. Since (5.22) holds for all $N \in \mathbb{Z}$, it might be possible to connect (5.22) with some 3D lattice equations. Finally, let us go back to $x_{i}$ defined in (3.7). It is interesting that all the $\left\{\alpha_{N}\right\}$ can be expressed in terms of $x_{i}$. Recalling Lemma 3.4 in which $v_{N}$ can be positive in the first quadrant $\{n \geq 0, m \geq 0\}$ if we take $v_{N}(0,0)>0$ which can be done by suitably choosing value for $\gamma_{2 N-1}$ (see (3.6) as examples), we can make use of the relation between $v_{N}$ and $f$ to formulate a mechanism for choosing $\gamma_{j}$ so that $f$ is nonzero in the first quadrant. This will be done in Appendix C.

At the end of the paper we would like to make a comparison for the rational solutions and their derivation between the present paper and [21]. In this paper the construction of rational solutions is based on iteration of a chain of transformations, and the unified $\tau$ function $f(\alpha)=|\widehat{N-1}|$ is proved to satisfy the bilinear superposition formula (5.22). In [21], rational solutions (most of them with exponential background) for $\mathrm{H} 3(\delta)$ and $\mathrm{Q} 1(\delta)$ are obtained via a limiting procedure from soliton solutions in Casoratian expression. The method used in [21] can be extended to H1 and H2 (by selecting (5.9) as a basic Casoratian entry) and the results will be the same as the present paper. However, for $\mathrm{H} 3(\delta)$ and $\mathrm{Q} 1(\delta)$ it is obvious that our construction, which brings pure rational solutions, allows reduction $\delta=0$ and relies only on a unified $\tau$ function, has more advantage than the limiting procedure used in [21]. It is hard to say what is the reason of this difference, but a fact is all the BTs we used in our paper are only parametrically related to spacing parameters $a, b$ without any extra parameters for solitons. These BTs are natural for generating rational solutions.

\section{A Proof of Theorem 5.1 for $\mathrm{H} 1$}

Here we prove Theorem 5.1 which gives Casoratian form of rational solutions of H1.

First, we prove (5.8a). Noticing that $\psi_{i}$ defined in (5.9) satisfies shift relation

$$
\psi_{i}(l)-\underset{\sim}{\psi_{i}}(l+1)=(1-a) \underset{\sim}{\psi_{i}}(l)
$$


and $\psi_{i}\left(\right.$ with $\left.\varrho_{i}^{ \pm}(5.10)\right)$ and $\alpha_{j}$ defined by (5.13) actually obey the relation

$$
\psi_{i}(l)=\sum_{j=0}^{\infty} \alpha_{j}(l) s_{i}^{2 j+1}
$$

we have

$$
\alpha_{i}(l)-\underset{\sim}{\alpha} \alpha_{i}(l+1)=(1-a) \underset{\sim}{\alpha_{i}}(l) .
$$

With such a shift relation and using the technique in [14], for the Casoratians in (5.14) we find

$$
\begin{aligned}
& (1-a)^{N-1} \underset{\sim}{f}=|\widehat{N-2}, \underset{\sim}{\alpha}(N-1)|, \\
& -a(1-a)^{N-1} \underset{\sim}{\underset{\sim}{f}}=|\widehat{N-1}, \underset{\sim}{\alpha}(N-1)|, \\
& -a(1-a)^{N-1} \underset{\sim}{g}=|\widehat{N-2}, N \underset{\sim}{\alpha}(N-1)|+(1-a)^{N-1}(1+a N) \underset{\sim}{\bar{f}},
\end{aligned}
$$

where we have neglected subscript " $R$ " without making any confusion.

Substituting (5.14) and (A.3) into the downtilde-shifted (5.8a), for the l.h.s. we reach

$$
|\widehat{N}||\widehat{N-2}, \underset{\sim}{\alpha}(N-1)|-|\widehat{N-1}, \underset{\sim}{\alpha}(N-1)||\widehat{N-2}, N|+|\widehat{N-1}||\widehat{N-2}, N, \underset{\sim}{\alpha}(N-1)|,
$$

which is zero in light of Lemma 2.1. In fact, we can replace the $N$-th order vector $\alpha$ with $(N+1)$ th order one, introduce an auxiliary $(N+1)$-th order column vector $e_{N+1}=(0,0, \ldots, 0,1)^{\mathrm{T}}$, and rewrite

$$
\begin{aligned}
& f=\left|\widehat{N-1}, e_{N+1}\right|, \quad g=\left|\widehat{N-2}, N, e_{N+1}\right|, \\
& |\widehat{N-2}, \underset{\sim}{\alpha}(N-1)|=\mid \widehat{N-2}, \underset{\sim}{\alpha(N-1), e_{N+1} \mid}
\end{aligned}
$$

then after taking $\mathbf{B}=(\widehat{N-2}), \mathbf{a}=\alpha(N-1), \mathbf{b}=e_{N+1}, \mathbf{c}=\alpha(N), \mathbf{d}=\alpha(N-1),($ A.4 $)$ vanishes due to Lemma 2.1.

Next, to prove (5.8b) we consider Casoratians $f$ and $g$ composed by $\phi(l)=\left(\phi_{1}, \phi_{2}, \ldots, \phi_{N}\right)^{\mathrm{T}}$ where

$$
\phi_{i}(n, m, l)=\varrho_{i}^{+}\left(1+s_{i}\right)^{l}\left(1-a s_{i}\right)^{-n}\left(1+b s_{i}\right)^{m}+\varrho_{i}^{-}\left(1-s_{i}\right)^{l}\left(1+a s_{i}\right)^{-n}\left(1-b s_{i}\right)^{m},
$$

which satisfies

$$
\phi_{i}(l)+a \widetilde{\phi}_{i}(l+1)=(1+a) \widetilde{\phi}_{i}(l) .
$$

Introduce vector

$$
\omega(l)=\left(\omega_{1}(l), \omega_{2}(l), \ldots, \omega_{N}(l)\right)^{\mathrm{T}}, \quad \omega_{j}=\left.\frac{1}{(2 j+1) !} \partial_{s_{i}}^{2 j+1} \phi_{i}\right|_{s_{i}=0} .
$$

Noticing the expression (A.1) for $\alpha_{j}(l)$ and relation

$$
\phi_{i}=\frac{1}{\left(1-a^{2} s_{i}^{2}\right)^{n}} \psi_{i}
$$

where we have taken $\varrho_{i}^{ \pm}$defined as (5.10), we find

$$
\omega=A \alpha,
$$


where $A=\left(a_{i j}\right)_{N \times N}$ is a lower triangular Toeplitz matrix defined by

$$
a_{i j}= \begin{cases}0, & i<j \\ \left.\frac{\partial_{s_{i}}^{2(i-j)}}{[2(i-j)] !} \frac{1}{\left(1-a^{2} s_{i}^{2}\right)^{n}}\right|_{s_{i}=0}, & i \geq j\end{cases}
$$

Noticing the relation (A.6) and $|A|=1$, we have

$$
f(\omega(l))=|A| f(\alpha(l))=f(\alpha(l)), \quad g(\omega(l))=g(\alpha(l)) .
$$

Besides, $\omega_{i}$ obeys the same shift relation as (A.5), i.e.,

$$
\omega_{i}(l)+a \widetilde{\omega}_{i}(l+1)=(1+a) \widetilde{\omega}_{i}(l),
$$

which leads to

$$
\begin{aligned}
& (1+a)^{N-1} \widetilde{f}(\omega(l))=|\widehat{N-2}, \widetilde{\omega}(N-1)|, \\
& a(1+a)^{N-1} \widetilde{\bar{f}}(\omega(l))=|\widehat{N-1}, \widetilde{\omega}(N-1)|, \\
& a(1+a)^{N-1} \widetilde{\bar{g}}(\omega(l))=|\widehat{N-2}, N, \widetilde{\omega}(N-1)|-(1+a)^{N-1}(N a-1) \widetilde{\bar{f}} .
\end{aligned}
$$

Then one can find the 1.h.s. of (5.8b) yields

$$
|\widehat{N}||\widehat{N-2}, \widetilde{\omega}(N-1)|-|\widehat{N-1}, \widetilde{\omega}(N-1)||\widehat{N-2}, N|+|\widehat{N-1}||\widehat{N-2}, N, \widetilde{\omega}(N-1)|,
$$

which vanishes as (A.4).

(5.8c) and (5.8d) can be proved similarly.

\section{B Proof of Theorem 5.7 for $\mathrm{H2}$}

To prove Theorem 5.7, we rewrite

$$
h=s+t, \quad s=|\widehat{N-2}, N+1|_{\mathrm{R}}, \quad t=|\widehat{N-3}, N-1, N|_{\mathrm{R}} .
$$

With the relation (A.2) and using the technique in [14], for the Casoratians (5.26) we have

$$
\begin{aligned}
& a(1-a)^{N-2}\left[\underset{\sim}{s}+\left(a^{-1}-1\right)(\underset{\sim}{g}+N \underset{\sim}{f})\right]=-|\widehat{N-3}, N, \underset{\sim}{\alpha}(N-2)|, \\
& a(1-a)^{N-2}\left[\underset{\sim}{g}+\left(a^{-1}+N-1\right) \underset{\sim}{f}\right]=-|\widehat{N-3}, N-1, \underset{\sim}{\alpha}(N-2)|, \\
& a(1-a)^{N-2} \underset{\sim}{f}=-|\widehat{N-3}, N-2, \underset{\sim}{\alpha}(N-2)| .
\end{aligned}
$$

Again, here and after we drop off subscript " $R$ " without making any confusion. Then we find that

$$
\begin{aligned}
a(1-a)^{N-2}\left\{f\left[\underline{s}+\left(a^{-1}-1\right)(\underset{\sim}{g}+N \underset{\sim}{f})\right]-(g+N f)\left[\underset{\sim}{g}+\left(N+a^{-1}-1\right) \underset{\sim}{f}\right]+\underset{\sim}{f} t\right\} \\
=-|\widehat{N-1}||\widehat{N-3}, N, \underset{\sim}{\alpha(N-2)|+| \widehat{N-2}}, N||\widehat{N-3}, N-1, \underset{\sim}{\alpha}(N-2)| \\
\quad-|\widehat{N-3}, N-2, \underset{\sim}{\alpha}(N-2)||\widehat{N-3}, N-1, N|=0 .
\end{aligned}
$$

Since

$$
f(\omega(l))=f(\alpha(l)), \quad g(\omega(l))=g(\alpha(l)), \quad s(\omega(l))=s(\alpha(l)), \quad t(\omega(l))=t(\alpha(l)),
$$


using (A.7) one has

$$
\begin{aligned}
& a(1+a)^{N-2}\left[\widetilde{s}(\omega)-\left(a^{-1}+1\right)(\widetilde{g}(\omega)+N \widetilde{f}(\omega))\right]=|\widehat{N-3}, N, \widetilde{\omega}(N-2)|, \\
& a(1+a)^{N-2}\left[\widetilde{g}(\omega)-\left(a^{-1}-N+1\right) \widetilde{f}(\omega)\right]=|\widehat{N-3}, N-1, \widetilde{\omega}(N-2)|, \\
& a(1+a)^{N-2} \widetilde{f}(\omega)=|\widehat{N-2}, \widetilde{\omega}(N-2)| .
\end{aligned}
$$

Consequently it reaches

$$
\begin{aligned}
a(1+ & a)^{N-2}\left\{f\left[\widetilde{s}-\left(a^{-1}+1\right)(\widetilde{g}+N \widetilde{f})\right]-(g+N f)\left[\widetilde{g}-\left(a^{-1}-N+1\right) \widetilde{f}\right]+\widetilde{f} t\right\} \\
= & |\widehat{N-1}||\widehat{N-3}, N, \widetilde{\omega}(N-2)|-|\widehat{N-2}, N||\widehat{N-3}, N-1, \widetilde{\omega}(N-2)| \\
& +|\widehat{N-2}, \widetilde{\omega}(N-2)||\widehat{N-3}, N-1, N|=0 .
\end{aligned}
$$

Then, adding (B.2) and the uptilde-shifted (B.1) yields (5.25a). The other equation in (5.25) can be proved similarly.

\section{Property of $f$}

In the following we take a close look at Casoratian $f_{N}$ defined by (5.14a), i.e.,

$$
f_{N}=|\widehat{N-1}|=|\alpha(n, m, 0), \alpha(n, m, 1), \ldots, \alpha(n, m, N-1)|,
$$

where $\alpha$ is given by (5.13). Due to relation (5.23), we only consider the case $N \in \mathbb{Z}^{+}$. To investigate properties of $f_{N}$, we introduce "degree" for a polynomial. For a monomial $\prod_{i \geq 1} x_{i}^{k_{i}}$ where $x_{i}$ is defined in (3.7), we assign it a degree $\sum_{i \geq 1} i k_{i}$ and denote this number by $\mathcal{D}\left[\prod_{i \geq 1} x_{i}^{k_{i}}\right]$. A polynomial $P=P\left[\left\{x_{i}\right\}\right]$ in which each monomial has same degree $d$ is called homogeneous and its degree is denoted by $\mathcal{D}[P]=d$. Under this definition, for the $f_{N}$ given in (5.15), they are all homogeneous and their degrees are $\mathcal{D}\left[f_{1}\right]=0, \mathcal{D}\left[f_{2}\right]=3, \mathcal{D}\left[f_{3}\right]=6$. In particular, we have

Lemma C.1. For $\alpha_{h}^{+}(n, m, l)$ defined in (5.12), $\alpha_{h}^{+}(n, m, 0)$ is homogeneous with degree

$$
\mathcal{D}\left[\alpha_{h}^{+}(n, m, 0)\right]=h .
$$

Now we come to investigate properties of $f_{N}$.

Theorem C.2. Casoratian $f_{N}$ in which $\alpha$ is given by (5.13) has the following properties:

(i) $f_{N}$ is homogeneous with degree $\mathcal{D}\left[f_{N}\right]=\frac{N(N+1)}{2}$;

(ii) $f_{N}$ depends only on $\left\{x_{1}, x_{3}, \ldots, x_{2 N-1}\right\}$;

(iii) $f_{N}(n, m)$ is positive in the first quadrant $\{n \geq 0, m \geq 0\}$ provided $a>0, b>0$ and $f_{N}(0,0)>0$

(iv) in construction, $f_{N}(0,0)>0$ is guaranteed by successively choosing

$$
(-1)^{N+1} \gamma_{2 N-1}>-\frac{\left.(2 N-1) f_{N}(0,0)\right|_{\gamma_{2 N-1}=0}}{f_{N-2}(0,0)} .
$$

Proof. We prove the items of the theorem one by one.

(i) Consider Casoratian $f_{N}$ (C.1) in which $\alpha$ is given by (5.13). $f_{N}$ is written as $\left|\left(f_{i j}\right)_{N \times N}\right|$ where $f_{i j}=\alpha_{2 i-1}^{+}(n, m, j-1)$. Noting that it is not $\alpha_{h}^{+}(n, m, l)$ but $\alpha_{h}^{+}(n, m, 0)$ that is homogeneous, in the following we make use of shift relation (5.21) to rewrite $f_{N}$ in terms of $\left\{\alpha_{h}^{+}(n, m, 0)\right\}$. To do that, first from (5.21) we have

$$
\begin{aligned}
& \alpha(l+1)-\alpha(l)=\beta(l), \\
& \beta(l+1)-\beta(l)=\Lambda \alpha(l),
\end{aligned}
$$


where

$$
\Lambda=\left(\delta_{i, j+1}\right)_{N \times N}, \quad \delta_{i, j}= \begin{cases}1, & i=j, \\ 0, & i \neq j .\end{cases}
$$

Here in (C.2) we have omitted $n, m$ in $\alpha(n, m, l)$ and $\beta(n, m, l)$ for convenience. Successively making use of (C.2a) we can rewrite (C.1) from the last to the first column, and we have

$$
f_{N}=|\alpha(0), \beta(0), \beta(1), \beta(2), \ldots, \beta(N-3), \beta(N-2)| .
$$

Then, employing (C.2b) and in a similar manner we have

$$
f_{N}=|\alpha(0), \beta(0), \Lambda \alpha(0), \Lambda \alpha(1), \ldots, \Lambda \alpha(N-4), \Lambda \alpha(N-3)| .
$$

This procedure can be continued until we arrive at

$$
f_{N}=\left|\alpha(0), \beta(0), \Lambda \alpha(0), \Lambda \beta(0), \Lambda^{2} \alpha(0), \Lambda^{2} \beta(0), \ldots, \Lambda^{[(N-1) / 2]} \sigma(0)\right|,
$$

where

$$
\sigma(0)= \begin{cases}\alpha(0), & N \text { odd } \\ \beta(0), & N \text { even }\end{cases}
$$

(C.3) can be denoted by $f_{N}=\left|\left(f_{i j}^{\prime}\right)_{N \times N}\right|$ where

$$
f_{i j}^{\prime}= \begin{cases}\alpha_{2 i-j}^{+}(0), & i=1,2, \ldots, N, 1 \leq j \leq \min \{2 i, N\} \\ 0, & \min \{2 i, N\}<j \leq N\end{cases}
$$

Now it is evident that each element in (C.3) is homogenous with degree

$$
\mathcal{D}\left[f_{i j}^{\prime}\right]= \begin{cases}2 i-j, & i=1,2, \ldots, N, 1 \leq j \leq \min \{2 i, N\}, \\ \text { not available, } & \min \{2 i, N\}<j \leq N .\end{cases}
$$

Note that the degree of nonzero $f_{i j}^{\prime}$ is separable in terms of $i$ and $j$. Meanwhile, $f_{N}$ is an algebraic summation in which each term is a nonzero product $\prod_{i=1}^{N} f_{i, j_{i}}^{\prime}$ where $j_{i}$ runs over a permutation of the set $\{1,2, \ldots, N\}$. It is easy to get

$$
\mathcal{D}\left[\prod_{i=1}^{N} f_{i, j_{i}}^{\prime}\right]=\sum_{i=1}^{N}(2 i)-\sum_{j=1}^{N} j=\frac{N(N+1)}{2}
$$

which means $f_{N}$ is homogeneous with degree $\mathcal{D}\left[f_{N}\right]=\frac{N(N+1)}{2}$.

(ii) In the following we come to the statement that $f_{N}$ depends only on $\left\{x_{1}, x_{3}, \ldots, x_{2 N-1}\right\}$, which has been shown correct for $N=1,2,3$ in (5.15). Now we assume the statement is correct for $f_{j}$ where $j \in\{1,2, \ldots, N-1\}$. For $f_{N}$, first, it contains $x_{2 N-1}$. In fact, from the expression (C.3) we can see that $x_{2 N-1}$ only appears in the element $f_{N, 1}^{\prime}$, and doing Laplace expansion for (C.3) along the first two columns it is easy to find the only term involving $x_{2 N-1}$ is

$$
\frac{(-1)^{N+1}}{2 N-1} x_{2 N-1} f_{N-2} \text {. }
$$

Next, we note that for each monomial $A=\prod_{i \geq 1} x_{i}^{j_{i}}$, the commutating relation

$$
\partial_{x_{i}} \widetilde{A}=\widetilde{\partial_{x_{i}} A}
$$


holds, which indicates $\partial_{x_{i}} \widetilde{f}_{N}=\widetilde{\partial_{x_{i}} f_{N}}$. Taking derivative $\partial_{x_{2 i}}$ on both sides of double down bar shifted $(5.22 \mathrm{a})$ we have

$$
f_{N-2} \widetilde{\partial_{x_{2 i}} f_{N}}-\widetilde{f}_{N-2} \partial_{x_{2 i}} f_{N}=0
$$

where the right hand side has vanished due to the assumption that $f_{j}$ is independent of $x_{2 i}$ for $j \in\{1,2, \ldots, N-1\}$. The above relation indicates $\frac{\partial_{x_{2}} f_{N}}{f_{N-2}}$ is independent of $n$. In a same manner, from $(5.22 \mathrm{~b})$ we can find $\frac{\partial_{x_{2 i}} f_{N}}{f_{N-2}}$ is independent of $m$. Thus we come to a relation

$$
\partial_{x_{2 i}} f_{N}=c f_{N-2}
$$

where $c$ is a constant. Based on item (i) of the current theorem the degrees in (C.5) read

$$
\frac{N(N+1)}{2}-2 i=\frac{(N-2)(N-1)}{2},
$$

i.e.,

$$
2 N-1=2 i
$$

which is contradictory for any $i \in \mathbb{Z}^{+}$and then indicates $c=0$ in (C.5). Thus, $\partial_{x_{2 i}} f_{N}=0$, i.e., $f_{N}$ is independent of $x_{2 i}$. In conclusion, $f_{N}$ depends only on $\left\{x_{1}, x_{3}, \ldots, x_{2 N-1}\right\}$.

(iii) It is known that $v_{N}=f_{N} / f_{N-2}$ and $V_{N}=f_{N-1} / f_{N-2}$ provide a solution pair to (3.3b). Meanwhile, from Lemma 3.4 both $v_{N}$ and $V_{N}$ are positive in the first quadrant $\{n \geq 0, m \geq 0\}$ if $a>0, b>0$ and $v_{N}(0,0)>0$. Obviously, from $v_{N}=f_{N} / f_{N-2}$ and $f_{-1}=f_{0}=1$, for each $N=2,3, \ldots$, we can successively find $f_{N}(n, m)>0$ in the first quadrant if $a>0, b>0$ and $f_{N}(0,0)>0$.

(iv) Finally, we formulate a mechanism to guarantee $f_{N}(0,0)>0$ by choosing suitable $\gamma_{j}$. From item (ii) we know that $f_{N}(0,0)$ is only related to $\left\{\gamma_{1}, \gamma_{3}, \ldots, \gamma_{2 N-1}\right\}$. Since in $f_{N}$ the term involving $x_{2 N-1}$ is (C.4), we express $f_{N}$ as

$$
f_{N}(n, m)=\left.f_{N}(n, m)\right|_{x_{2 N-1}=0}+\frac{(-1)^{N+1}}{2 N-1} x_{2 N-1} f_{N}(n, m),
$$

which, at point $(0,0)$, yields

$$
f_{N}(0,0)=\left.f_{N}(0,0)\right|_{\gamma_{2 N-1}=0}+\frac{(-1)^{N+1}}{2 N-1} \gamma_{2 N-1} f_{N-2}(0,0) .
$$

To guarantee $f_{N}(0,0)>0$, we need to take

$$
(-1)^{N+1} \gamma_{2 N-1}>-\frac{\left.(2 N-1) f_{N}(0,0)\right|_{\gamma_{2 N-1}=0}}{f_{N-2}(0,0)} .
$$

Thus, as a starting step we take $f_{0}=1$ and $f_{1}=x_{1}$ with $\gamma_{1}>0$, for $N=2$, using the above formula we choose a value for $\gamma_{3}$ so that $f_{2}(0,0)>0$. We can repeatedly use (C.6) and will successively choose $\gamma_{2 N-1}$ and get $f_{N}(0,0)>0$ for higher $N$.

\section{Acknowledgements}

We are grateful to the referee for the invaluable comments. This project is supported by the NSF of China (no. 11371241 and no. 11631007). 


\section{References}

[1] Ablowitz M.J., Satsuma J., Solitons and rational solutions of nonlinear evolution equations, J. Math. Phys. 19 (1978), 2180-2186.

[2] Adler V.E., Bobenko A.I., Suris Yu.B., Classification of integrable equations on quad-graphs. The consistency approach, Comm. Math. Phys. 233 (2003), 513-543, nlin.SI/0202024.

[3] Adler V.E., Bobenko A.I., Suris Yu.B., Discrete nonlinear hyperbolic equations: classification of integrable cases, Funct. Anal. Appl. 43 (2009), 3-17, arXiv:0705.1663.

[4] Atkinson J., Bäcklund transformations for integrable lattice equations, J. Phys. A: Math. Theor. 41 (2008), 135202, 8 pages, arXiv:0801.1998.

[5] Atkinson J., Hietarinta J., Nijhoff F.W., Seed and soliton solutions for Adler's lattice equation, J. Phys. A: Math. Theor. 40 (2007), F1-F8, nlin.SI/0609044.

[6] Atkinson J., Hietarinta J., Nijhoff F.W., Soliton solutions for Q3, J. Phys. A: Math. Theor. 41 (2008), 142001, 11 pages, arXiv:0801.0806.

[7] Atkinson J., Nijhoff F.W., A constructive approach to the soliton solutions of integrable quadrilateral lattice equations, Comm. Math. Phys. 299 (2010), 283-304, arXiv:0911.0458.

[8] Bobenko A.I., Suris Yu.B., Integrable systems on quad-graphs, Int. Math. Res. Not. 2002 (2002), 573-611, nlin.SI/0110004.

[9] Feng W., Zhao S., Shi Y., Rational solutions for lattice potential KdV equation and two semi-discrete lattice potential KdV equations, Z. Natur. A 71 (2016), 121-128.

[10] Freeman N.C., Nimmo J.J.C., Soliton solutions of the Korteweg-de Vries and Kadomtsev-Petviashvili equations: the Wronskian technique, Phys. Lett. A 95 (1983), 1-3.

[11] Grammaticos B., Ramani A., Papageorgiou V., Satsuma J., Willox R., Constructing lump-like solutions of the Hirota-Miwa equation, J. Phys. A: Math. Theor. 40 (2007), 12619-12627.

[12] Hietarinta J., Boussinesq-like multi-component lattice equations and multi-dimensional consistency, J. Phys. A: Math. Theor. 44 (2011), 165204, 22 pages, arXiv:1011.1978.

[13] Hietarinta J., Joshi N., Nijhoff F.W., Discrete systems and integrability, Cambridge Texts in Applied Mathematics, Cambridge University Press, Cambridge, 2016.

[14] Hietarinta J., Zhang D.-J., Soliton solutions for ABS lattice equations. II. Casoratians and bilinearization, J. Phys. A: Math. Theor. 42 (2009), 404006, 30 pages, arXiv:0903.1717.

[15] Hirota R., Nonlinear partial difference equations. I. A difference analogue of the Korteweg-de Vries equation, J. Phys. Soc. Japan 43 (1977), 1424-1433.

[16] Ma W.-X., You Y., Solving the Korteweg-de Vries equation by its bilinear form: Wronskian solutions, Trans. Amer. Math. Soc. 357 (2005), 1753-1778, nlin.SI/0603008.

[17] Maruno K., Kajiwara K., Nakao S., Oikawa M., Bilinearization of discrete soliton equations and singularity confinement, Phys. Lett. A 229 (1997), 173-182, solv-int/9610005.

[18] Nijhoff F.W., Lax pair for the Adler (lattice Krichever-Novikov) system, Phys. Lett. A 297 (2002), 49-58, nlin.SI/0110027.

[19] Nijhoff F.W., Atkinson J., Hietarinta J., Soliton solutions for ABS lattice equations. I. Cauchy matrix approach, J. Phys. A: Math. Theor. 42 (2009), 404005, 34 pages, arXiv:0902.4873.

[20] Nijhoff F.W., Walker A.J., The discrete and continuous Painlevé VI hierarchy and the Garnier systems, Glasg. Math. J. 43A (2001), 109-123, nlin.SI/0001054.

[21] Shi Y., Zhang D.-J., Rational solutions of the H3 and Q1 models in the ABS lattice list, SIGMA 7 (2011), 046, 11 pages, arXiv:1105.1583.

[22] Zhang D.-J., Hietarinta J., Generalized solutions for the H1 model in ABS list of lattice equations, in Nonlinear and Modern Mathematical Physics, AIP Conf. Proc., Vol. 1212, Amer. Inst. Phys., Melville, NY, 2010, 154-161. 